\title{
The influence of predation risk on diet selectivity: A theoretical analysis
}

\author{
ROBERT HOUTMAN* and LAWRENCE M. DILL \\ Behavioural Ecology Research Group, Department of Biological Sciences, Simon Fraser University, Burnaby, British Columbia \\ V5A 1S6, Canada
}

\begin{abstract}
Summary
Studies that have examined the effect of experimental increases in predation risk on diet selectivity have shown both decreased and increased diet selectivity. A possible explanation for these disparate results emerges from an examination of the prey sets used in these studies, which differed in the relationship between the values of risk components associated with the capture of different prey types ('danger') and their profitabilities. When less profitable prey were more dangerous, selectivity increased with predation risk. When prey were equally dangerous, selectivity did not change. Finally, when the more profitable prey were also more dangerous, selectivity decreased with risk. Here, we examine theoretically the influence of a forager's estimate of the probability that a predator is present $(\phi)$ on the selection of diets from prey sets with varying dangerprofitability relationships. A dynamic programming model is used to determine the maximum attack time (or distance) for each of two types of prey, differing in their energetic content, for a range of forager energy state and $\phi$ levels. The diets which would result if foragers attacked prey according to the rules provided by the dynamic model are then determined. The model results indicate that the prey danger-profitability relationship determines the diet selectivity response to $\phi$, confirming that variation in this relationship could be responsible for the range of experimental results.
\end{abstract}

Keywords: crypsis; diet selectivity; dynamic programming; foraging; modelling; predation risk

\section{Introduction}

The prediction and explanation of animal diet selectivity is an important research topic in behavioural ecology. First-generation studies of this problem considered only the energetic benefittime cost trade-off (Stephens and Krebs, 1986). Recently, studies have begun to investigate the importance of other, higher-level trade-offs. One such trade-off is that between foraging benefits (net energy intake) and predation risk costs. Prey capture actions will have an associated predation risk cost in many situations. For example, an animal's vigilance is often compromised when attacking and/or handling prey, decreasing the probability that the animal will detect an attacking predator (Milinski, 1984), and presumably resulting in reduced chances of escape (see Godin and Smith, 1988). A second example concerns cryptic animals; for them, prey capture movements may reduce or completely eliminate their crypsis, increasing the probability of detection by predators and, thus, predation risk (Edmunds, 1974; Morey, 1990).

The term 'predation risk' has several different meanings. Here, the predation risk of a given action is the probability of death by predation during the execution of the action, and is the product of the probabilities of the components of predation risk. These components correspond to the steps leading to an animal's death by predation: a predator is present (close enough to detect and attack the animal), it detects the animal, attacks the animal, and captures the animal. (For

\footnotetext{
*Author to whom all correspondence should be addressed.
} 
simplicity, we assume that predators attack immediately upon detection of prey; thus, the predation risk after completion of an action is not influenced by that action.) An animal will often not be certain whether or not a predator is present, but will have an estimate of the probability that one is, based on the information it has. We refer to the animal's estimate of this probability as $\phi$. Many authors use 'predation risk' synonymously with $\phi$; however, this can lead to confusion and hinder new insights regarding the effects of predation risk. We refer to the probabilities of each of the final three steps as $\operatorname{Pr}$ (detection), $\operatorname{Pr}$ (attack) and $\operatorname{Pr}$ (capture), respectively (note that these three probabilities are all conditional on the steps earlier in the sequence).

Typical tests of whether predation risk influences diet selectivity involve determining subjects' diet selectivities in two treatments: (1) high $\phi$, where a subject's $\phi$ is elevated to close to 1.0 by presenting a predator or predator cue during a trial; and (2) low $\phi$, achieved by having no predator cue present. We use the term 'diet selectivity response' to refer to the difference in diet selectivity at high minus low overall risk.

To date, four studies have measured diet selectivity responses. Godin (1990) studied guppies (Poecilia reticulata) foraging on Daphnia of three different size classes. He found that the guppies' handling times and attack success on the three sizes differed depending on whether a predator was present. However, the profitabilities (including the time and energy cost of failed capture attempts) were probably greatest for small Daphnia, least for large ones and intermediate for the medium-size class both when a predator was and was not present. During trials with a predatory fish visible to the subjects (high $\phi$ ), guppies captured relatively more small, profitable prey than in trials with no predator present (low $\phi$ ); thus, in this study, the diet selectivity response was positive - the guppies became more selective.

In a similar study, Ibrahim and Huntingford (1989) determined the diet selectivities of threespined sticklebacks (Gasterosteus aculeatus) foraging on chironomids and Bosmina, with and without a predatory fish visible. For sticklebacks, Bosmina are less profitable than chironomids (Ibrahim and Huntingford, 1989). Subjects in the predator-present treatment ate a larger proportion of Bosmina, and thus were less selective than control subjects (i.e. a negative diet selectivity response).

Houtman (1995) determined the diet selectivities of tidepool sculpins (Oligocottus maculosus) foraging on two size classes of brine shrimp (Artemia), with and without conspecific skin extract (to which these fish are known to show an alarm response; Hugie et al., 1991) present in the water. For sculpins, the larger size class of brine shrimp is more profitable. Houtman (1995) found no effect of skin extract on the proportion of the two prey size classes captured, and thus on diet selectivity (i.e. a null diet selectivity response).

Lima (1988) examined the diets of dark-eyed juncos (Junco hyemalis) foraging on whole millet seeds and millet 'bits'. In this study, Lima compared the diets of individuals foraging in different flock sizes, reasoning that 'an increase in group size effectively reduces an individual's risk of predation because other flock members are also vigilant, and the chance of death in the event of a successful attack decreases with group size' (Lima, 1988, p. 594). Thus, his comparisons of diet selectivities of birds in different group sizes can be viewed as comparisons at different predation risks (with risk increasing with decreasing flock size). He found that the proportion of whole seeds in the diets increased with decreasing flock size. Since the seeds were less profitable than the bits, diet selectivity decreased with decreasing flock size (increasing risk), a negative response. Lima's study was unique in two regards. First, he determined diets at 10 different flock size classes, and found that selectivity decreased continuously with decreasing flock size. Secondly, for each flock size, he determined diets for birds foraging on three different bit-whole seed pairings, distinguished by the size of bits (bit profitability increases with bit size). He found that at all flock sizes, diet selectivity (proportion of bits in diet) increased with bit size. Furthermore, he found that there was 
'a significant interaction between the effects of flock size and bit size; the effect of flock size was progressively smaller as bit size increased' (Lima, 1988, p. 595). Thus, the diet selectivity response (to a given decrease in flock size) decreased with increasing bit size, although it was always negative.

In summary, the results of these studies include positive, null and negative diet selectivity responses to manipulations of overall predation risk. These studies used different species of foragers and prey, and manipulated different predation risk components. Thus, there are several potential causes for the range of results. However, the variable most probably responsible is the relationship between the 'dangers' associated with capturing prey and their profitabilities.

In Godin's (1990) study, handling time increased with size of Daphnia. Therefore, he argued that larger prey were more dangerous to capture, because the prey handling probably reduced the guppies' ability to detect attacking predators. Since larger prey were also less profitable, the slope of the prey danger versus profitability relationship ('DP slope') was negative in Godin's study.

For sculpins, the major predation risk component elevated by prey capture is most likely $\operatorname{Pr}($ detection), as these animals are relatively cryptic when immobile (Houtman and Dill, 1994). In the experiment on sculpins, the two prey size classes had similar handling times (Houtman, 1995) and thus were probably equally dangerous to capture. Therefore, the sculpins were presented with a null DP slope (or close to null).

In the study of Ibrahim and Huntingford (1989), attack of the more profitable chironomids probably had higher associated values of Pr(capture) than did Bosmina (thus producing a positive DP slope) for two reasons. First, it is likely that prey handling reduces the probability of sticklebacks' detecting attacking predators, and chironomids require much longer handling times. Also, 'feeding on benthic chironomids requires the sticklebacks to assume a head-down posture which reduces their chances of detecting predatory attacks' even further (Ibrahim and Huntingford, 1989, p. 52).

In Lima's (1988) study, the less profitable whole millet seeds were safer, because during handling to remove the shell, the juncos assumed a head-up posture and thus could scan for predators. In contrast, the entire handling time of bits (of all three sizes) was spent with the head down. Thus, the juncos were also presented with positive DP slopes. Because profitabilities of the bits increased with size, but their dangers were relatively constant (i.e. they had similar handling times), the three different seed-bit pairings represented different (positive) DP slopes.

To summarize, positive, null and negative diet selectivity responses have been found in experiments that presented foragers with prey falling on a negative, null and positive DP slope, respectively (Fig. 1). Such a relationship is intuitively reasonable, considering that in the experiments with both positive and negative responses, the foragers always biased their diets more towards safer prey in situations with elevated values of overall predation risk (Lima, 1988; Ibrahim and Huntingford, 1989; Godin, 1990); thus, foragers reduced their rate of energy gain in exchange for increased safety. Furthermore, in the experiment on sculpins, in which prey did not differ in danger, a change in diet selectivity would not have increased the sculpins' safety (although a decreased total prey capture rate, which was shown, would have).

In this paper, we use a dynamic programming model (see Mangel and Clark, 1988) to determine prey attack policies for a forager maximizing its probability of survival to the end of a foraging period, for a range of DP slopes. These policies specify the maximum attack times (or distances) at which two types of prey should be attacked, over a range of energy and $\phi$ levels. Simulation of foraging bouts adhering to these policies allows diet selectivities to be calculated. By comparing the selectivities at high and low $\phi$ levels, the diet selectivity response to an increase in $\phi$ is determined. In general, we use $\phi$ levels of 0 and 1.0, because they should approximate the levels perceived by subjects in experiments in which diet selectivities are measured in high (predator stimulus present) and low (no stimulus present) 'predation risk' treatments. 


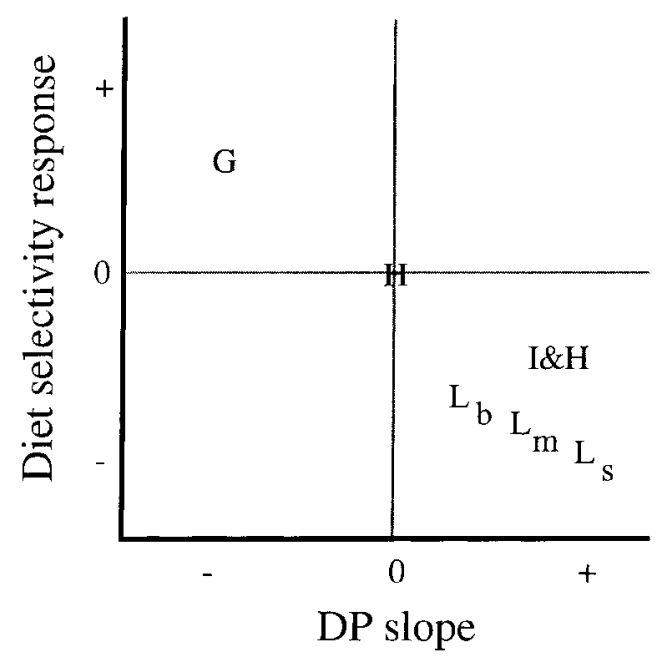

Figure 1. The diet selectivity responses to an increase in the value of overall predation risk versus the DP slope in the four experiments reviewed $(\mathrm{G}=$ Godin, 1990; $\mathrm{H}=$ Houtman, 1995; I\&H = Ibrahim and Huntingford, 1989; $\mathrm{L}=\mathrm{Lima}, 1988$ ). The plot is intended only to indicate the positions of the points relative to the origin. Although the precise nature of the relationship is not known, the three points from Lima's study (s, $\mathrm{m}$ and $\mathrm{b}$ indicate pairings consisting of seeds and small, medium and big bits, respectively) suggest that the diet selectivity response should change continuously with DP slope. Note that in Lima's study, selectivities were obtained at a whole range of flock sizes (i.e. overall predation risk levels); the responses shown represent the relative change in diet selectivity for any change in flock size in the three millet seed-bit pairings.

\section{The dynamic programming model}

Here, we present an overview of the dynamic programming model used to determine prey attack policies; the dynamic programming equation is described in the Appendix.

The dynamic programming model considers a forager feeding on two types of prey, differing in their gross energy content, during a foraging bout made up of discrete time periods. Prey type I has a higher energy content than type II. Both prey types can be encountered at any of four attack times (or distances), corresponding to 1,2,3 and 4 time periods. When a prey item is encountered, the forager has the option to 'accept' it (i.e. attack it and, it is assumed, capture it with probability $=1.0$ ) or 'reject' it. The dynamic model determines which of these two options maximizes the probability of survival from the current time period to the end of the foraging bout, for each prey type-attack time combination. This is done for each possible combination of values for two state variables, which describe the forager's energy and $\phi$ states.

The forager's energy state $(E(t))$ can vary over 25 levels. Unless prey are attacked, the forager's energy state decreases one level each time period, due to metabolic costs; if $E(t)$ reaches the lowest level, the forager dies of starvation. It is this feature which provides the 'impetus' for the forager to attack prey. When prey are attacked, the energy state is incremented by the gross energy content of the prey item minus the metabolic cost of attack (equal to one energy state level per time period). Note that this last feature makes the profitability of a prey attack dependent not only on the prey type attacked, but also on its attack time.

The information state at time $t$, denoted $\phi(t)$, represents the forager's assessment of $\phi$ in period $t$. The value of this variable changes as the forager gains information from scanning for predators and from not being captured by a predator (Houtman, 1995). The $\phi$ state has nine discrete levels, the 
lowest representing $\phi=0$ (certainty that no predator is present) and the highest $\phi=1.0$ (certainty that a predator is present). The value $\phi=1.0$ applies during a period immediately after predator detection; the lowest level is only reached after several consecutive periods without predator detection (see Houtman, 1995, for a complete description of the information state dynamics).

All of the activities which the forager can perform have associated predation risks. These are calculated as the sum of the probabilities of dying in each time period of an action. The probability of death in a particular time period, in turn, equals the product of $\operatorname{Pr}$ (alive at start of period), the $\phi$ which applies during that time period, $\operatorname{Pr}$ (detection), $\operatorname{Pr}($ attack) and $\operatorname{Pr}$ (capture). Houtman (1995) describes the predation risk calculations completely, and also lists the predation risk values corresponding to the run here with baseline parameter values (Table 1) and equal prey dangers.

We use $\operatorname{Pr}($ capture $\mid \mathrm{pp}$ ) (where 'pp' represents 'predator present') to refer to the product of $\operatorname{Pr}$ (detection), $\operatorname{Pr}$ (attack) and $\operatorname{Pr}$ (capture). Here, we vary the value of $\operatorname{Pr}$ (capture $\mid \mathrm{pp}$ ) to vary the 'danger' of different actions. $\operatorname{Pr}($ capture $\mid \mathrm{pp})$ is relatively low when the forager is not attacking a prey item, modelling (for example) a cryptic forager for whom $\operatorname{Pr}$ (detection) when not moving $<\operatorname{Pr}$ (detection) when attacking prey. In the present version, however, the value of $\operatorname{Pr}$ (capture $\mid \mathrm{pp}$ ) associated with attacking the two prey types can be varied to manipulate the DP slope. The energetic content of the two prey types, and thus their profitabilities at any given attack time, are held constant in all runs, making the DP slopes dependent only on the relative dangers of attack associated with attacking prey types I and II. Since the profitabilities of the two prey types vary with attack time, actual danger-profitability slopes cannot be defined. We use the difference in

Table 1. The dynamic programming model parameters (other than prey dangers; see Table 2) and their baseline values

Parameter

Baseline value

Prey parameters

Energy content of prey (in energy state units)

prey type I

prey type II

Attack times at which both prey types can be encountered (in time periods)

Probabilities of encountering prey at attack times of 1-4

(same for both prey types)

Probability of encountering no prey

\section{Forager parameters}

Metabolic rate (energy state units per time period)

$E(t)$ at which forager starves $\left(E_{\text {crit }}\right)$

5

3

$1,2,3,4$

$0.04,0.08,0.12,0.16$

0.2

Maximum $E(t)$

Number of information state levels

9

$\operatorname{Pr}$ (forager detects predator $\mid$ predator present)*

0.5

$\operatorname{Pr}($ capture $\mid \mathrm{pp}$ ) when not attacking prey

0.01

\section{Predator parameters}

$\begin{array}{ll}\operatorname{Pr}(\text { predator arrives } \mid \text { no predator present)* } & 0.05\end{array}$

$\operatorname{Pr}$ (predator leaves $\mid$ predator present)*

0.4

\footnotetext{
* For an explanation of these parameters, see Houtman (1995).
} 
$\operatorname{Pr}($ capture|pp) for the two prey types (prey type I minus prey type II) as an index of the DP slope. This index has the following desirable properties: (1) the difference is negative when the energetically richer prey (type I) is safer, and positive in the opposite situation; and (2) larger values represent steeper slopes, either negative or positive, while a value of zero represents the situation where prey have equal dangers.

The dynamic programming model was run with a variety of different DP slopes (Table 2), holding all other parameters constant. In each case, the model was run (backwards through time; see Mangel and Clark, 1988) until the decision matrix became stationary (defined as 20 time periods over which the decision matrix did not change). We determined diet selectivities for foragers at particular $\phi$ levels from these decision matrices.

\section{Obtaining diet selectivities}

The dynamic programming model provides decision matrices which specify maximum attack times at which the two prey types should be attacked, for all combinations of $E$ and $\phi$. We use a simulation procedure to determine the diet selectivities that result when an animal forages according to these rules.

The procedure simulates foraging bouts which are terminated when 10 prey have been attacked or the forager's energy state reaches $E_{\text {crit }}$ (and it starves to death). In the simulation, 'foragers' begin a foraging 'bout' with $E(t)=10$. An encounter type $i$ (no prey or one of the eight prey typeattack time combinations) is chosen randomly. If no prey is encountered, the energy state is decreased by one. If a prey item is encountered, the appropriate cell of the decision matrix is consulted to determine whether that prey class is to be accepted or rejected. If the prey is rejected, the energy state is decreased by one. If that prey class is to be accepted, the energy state is increased by the prey item's gross energy content minus 1 unit per handling time period. The forager's $\phi$ level is held constant for the duration of a foraging bout.

To provide a relatively stable estimate of diet selectivities, the program continues simulating foraging bouts until a total of 5000 prey have been attacked. Using the total number of each prey type (I and II) encountered and attacked during all of the simulated foraging bouts, a selectivity value is calculated using the formula for Chesson's $\alpha$ (Chesson, 1978); that is, selectivity $=\left(r_{\mathrm{I}} / p_{\mathrm{I}}\right) /\left[\left(r_{\mathrm{I}} / p_{\mathrm{I}}\right)+\left(r_{\mathrm{II}} / p_{\mathrm{II}}\right)\right]$, where I and II indicate the two prey types and $r_{i}$ and $p_{i}$

Table 2. The combinations of prey-specific $\operatorname{Pr}($ capture|pp) values ('dangers') and the resulting dangerprofitability slopes* used in model runs reported here

\begin{tabular}{lcc}
\hline Prey type I danger & Prey type II danger & DP slope \\
\hline 0.1 & 0.1 & 0 \\
0.075 & 0.125 & -0.05 \\
0.125 & 0.075 & +0.05 \\
0.05 & 0.15 & -0.1 \\
0.15 & 0.05 & +0.1 \\
0.025 & 0.175 & -0.15 \\
0.175 & 0.025 & +0.15 \\
0.0 & 0.2 & -0.2 \\
0.2 & 0.0 & +0.2 \\
\hline
\end{tabular}

* See text for an explanation of the DP slope calculation. 
represent the proportions of prey type $i$ eaten and encountered, respectively. To test the variation in selectivities, we ran the simulation twice for both the $\phi=0$ and $\phi=1.0$ levels of the decision matrices, for seven different DP slope situations. These 14 pairs of selectivities had a mean difference of 0.0023 (S.E. $=0.0013$ ), indicating very low variance.

Although this procedure calculates diet selectivities of a forager experiencing no change in $\phi$ during a foraging bout, the prey attack rules provided by the dynamic programming model represent the optimal decisions for the given $\phi$ level, when $\phi$ is a dynamic variable. Thus, the diet selectivities obtained in this way will represent those for foragers who 'expect' $\phi$ to change in the future.

\section{Simulation results and discussion}

The predicted relationship between diet selectivity and $\phi$, for danger-profitability (DP) slopes ranging from -0.15 to 0.15 , is shown in Fig. 2. With a slope of 0 , diet selectivity remains relatively constant with increasing $\phi$. When slopes are negative, diet selectivities generally increase with $\phi$, although a slight decrease is shown at higher $\phi$ values for DP slope $=-0.05$. Similarly, for positive DP slopes, selectivities decrease (at a decelerating rate) with increasing $\phi$ values. Furthermore, for any given $\phi$ level, selectivities decrease with increasing DP slope. Note that these two features, for the three positive DP slope curves, are found in Lima's (1988) results (discussed above), although in his study $\phi$ was not used to manipulate overall predation risk.

Figure 3 shows the relationship between the diet selectivity response and the DP slope. These responses equal the selectivity at $\phi=1.0$ minus that at $\phi=0$. The overall negative relationship, with a near-zero $y$-intercept, is as expected from the empirical evidence reviewed in the Introduction. This graph also indicates that the relationship is relatively continuous, although for DP slope $\leq-0.1$ and $\geq 0.1$, the response appears to be relatively independent of the DP slope.

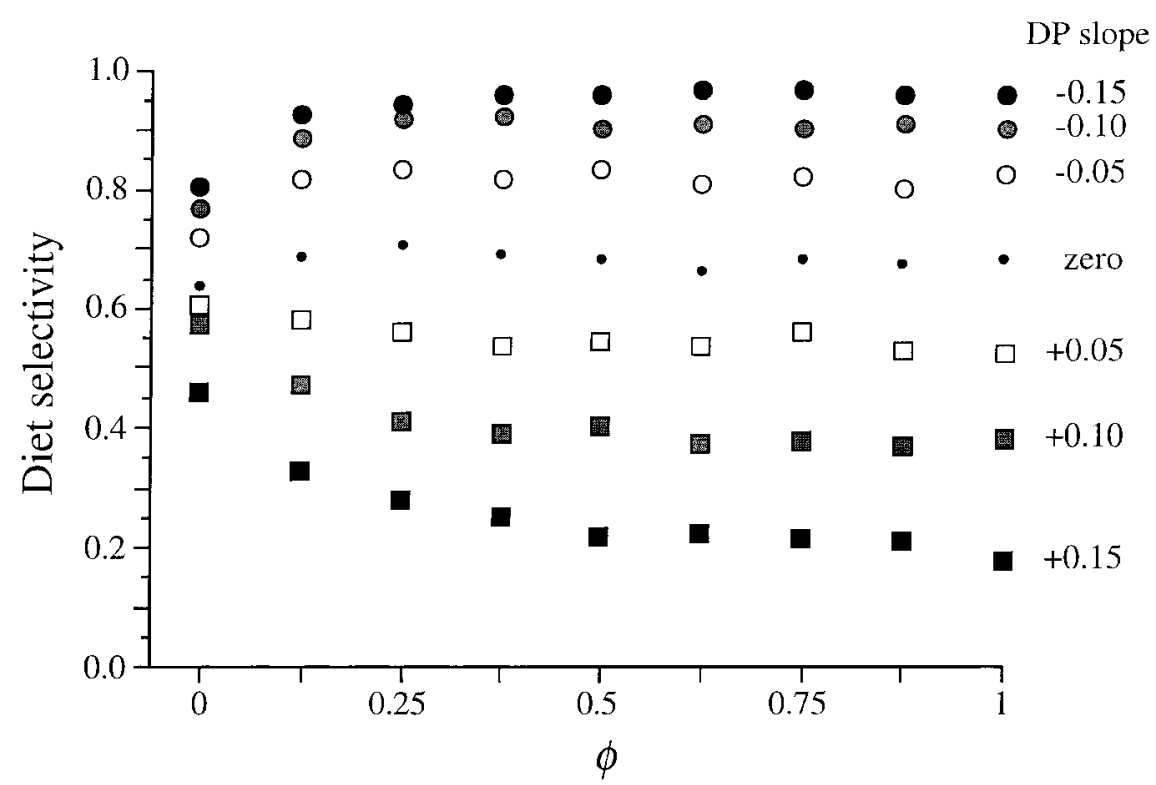

Figure 2. The predicted relationship between diet selectivity and $\phi$, the forager's perceived probability that a predator is present, for DP slopes ranging from -0.15 to +0.15 . 


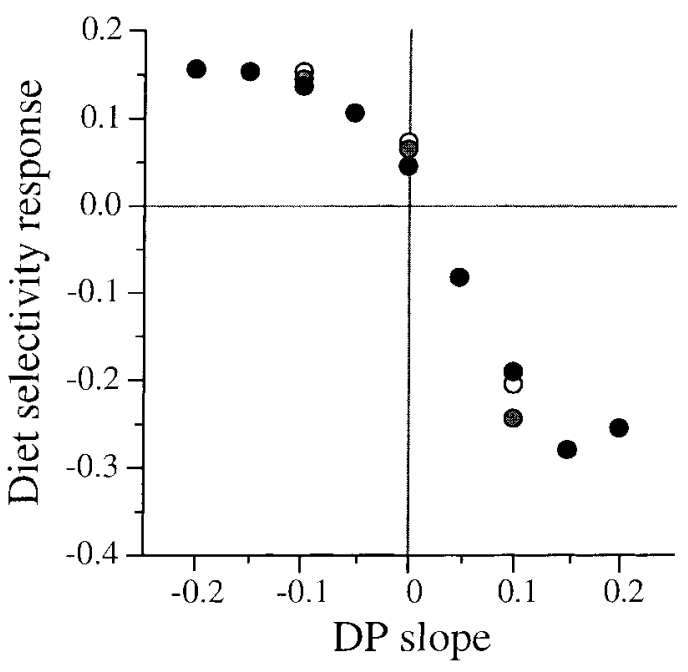

Figure 3. The relationship between diet selectivity response and DP slope for responses calculated using selectivities for $\phi=0$ and 1.0 (see text). Black circles represent values based on decision matrices produced by model runs using the baseline parameter set (Table 1). Grey and white circles represent values produced using parameter sets identical to the baseline set except that $\operatorname{Pr}($ predator arrives $\mid$ no predator present $)=0.1$ and $\operatorname{Pr}($ forager detects predator $\mid$ predator present $)=0.25$, respectively.

Finally, the two extra series plotted on the graph, for runs with $\operatorname{Pr}($ arrive $)=0.1$ and $\operatorname{Pr}(\operatorname{scan})=0.25$ (double and half the baseline values, respectively), indicate that the result is relatively robust to changes in these variables.

While the model predicts a slightly positive diet selectivity response at a DP slope of zero for all parameter sets tested, we believe this result to be a consequence of the limited number of discrete prey attack time options. Examination of the decision matrix for the zero DP slope situation indicates that prey type I is generally attacked at longer attack times (resulting in selectivities above 0.5 at all $\phi$ values; Fig. 2). This is reasonable, since prey type I is the more profitable one. At $\phi=1$, neither prey is attacked at $E(t)>9$ (for the baseline parameter set), because at these energy states the forager is better off waiting until $\phi$ decreases before capturing prey. At energy states of 8 and 9 , prey type I has a $\tau_{\max }$ (maximum attack time at which it should be accepted) of 1 , while that for prey type II is zero. Again, this is as expected given the equal prey dangers, and the constraint that prey can only be encountered at discrete attack times. However, this disparity (between $\tau_{\max }(\mathrm{I})=1$ and $\tau_{\max }(\mathrm{II})=0$ ) is probably greater than the optimal one at those energy states for a situation with continuous $\tau_{\max }$ options, and causes diet selectivities to be greater than those that would be predicted by a continuous model.

Note that this problem is not limited to cases where $\tau_{\max }(\mathrm{I}): \tau_{\max }(\mathrm{II})=1: 0$; however, at this ratio, the effect will be more severe than at less extreme ratios. This problem is also not limited to the run with DP slope $=0$. Similar extreme ratios occur in all decision matrices when $\phi \geq 0.125$, although they are reversed (prey type II remains in the diet after type I has been dropped) when the DP slope is positive. Thus, the actual effect of $\phi$ on diet selectivity will be less severe than indicated in Fig. 2, and the relationship between the selectivity response and the DP slope (Fig. 3) is expected to be less steep, with an intercept closer to zero. 


\section{General discussion}

The results support the role of the prey danger-profitability relationship (DP slope) on the diet selectivity response of a forager to an increase in overall predation risk. Viewed more generally, this result is equivalent to the results of most studies which have investigated foraging-predation risk trade-offs (reviewed in Lima and Dill, 1990); when overall predation risks are elevated (e.g. because of increased $\phi$, during exposure to a predator for instance), foragers tend to accept reduced energetic intake rates for reduced predation risk. For many foraging decisions, such as when or where to feed (cf. Lima and Dill, 1990), the behavioural option with the highest energetic return often has the highest associated predation risk. The case of prey choice, considered here, is interesting in that there is no a priori reason why alternative prey must represent an energy-risk trade-off (only the positive slope represents a trade-off).

In his review of the literature on the influence of predation risk on diet selection, Godin (1990) came to a similar conclusion regarding the importance of the relationship between prey danger and profitability on diet selectivity. However, his conclusion is stated more specifically, regarding only cases where vigilance and prey handling are incompatible, and handling time increases with prey size. Indeed, this influence of prey size on the probability of detecting a predator attack, and thus on $\operatorname{Pr}$ (capture), may be a common cause of prey-specific dangers. However, the realization that the value of other predation risk components may vary with prey type reveals the more general nature of the hypothesis.

Three other studies have examined the influence of predation risk on diet selectivity theoretically. Gilliam (1990) determined diet selectivity for foragers minimizing ' $\mu / f$ ' (the ratio of mortality rate to energy intake rate), when the predation risks of handling different prey types and of searching can differ. His analysis involved incorporating predation risks of search and handling into the basic prey model (for a review, see Stephens and Krebs, 1986). Gilliam (1990) compared diet breadths predicted by the basic prey model (with the optimization criterion 'maximize $f$ ') to those of his modified version.

Applying that model to the problem addressed here, that of predicting shifts in diets with changes in the probability that a predator is present, would require running it with two sets of predation risk values (for search and handling of all prey types), one with low values representing a low $\phi$ situation and one with higher values representing a high $\phi$ situation. The most reasonable assumption for assigning the values in the high $\phi$ set would be to multiply all of the risk values in the low $\phi$ set by the same factor, representing the elevation in $\phi$. However, in this case, Gilliam's (1990) model would predict no change in diet selectivity with $\phi$, regardless of difference in prey attack dangers. This is because the $\mu$ values for all possible diet breadths would increase by the same factor, and the diet minimizing $\mu / f$ would not change.

Gilliam (1990) cautioned 'that just comparing diets in the presence and absence of visible predators may not form a suitable test' of the model, since foragers may adjust parameters associated with prey (i.e. encounter probabilities, net energy gain, and/or handling time). Thus, the model would need to be run with different parameter sets to make appropriate predictions. In the current model, such an adjustment (in attack distances of the two prey types) is the mechanism which allows diet selectivity to vary with $\phi$.

Newman et al. (1995) used a dynamic programming model to predict diet selectivity of ruminants grazing in a pasture consisting of clover and ryegrass. In their model, there was no predation risk associated with resting or ruminating, while grazing (either or both species) had an associated predation risk. They examined the influence of predation risk on diet selectivity by running the model with a range of predation risk values. Their model predicts that, as predation risk (of grazing) increases, the animal will spend less total time grazing and more time ruminating, and its 
'preferred' prey will change from clover to grass. This interesting result appears to be caused by the differential digestibilities of the two plants (Newman et al., 1995): clover is more digestible without rumination than grass. As the predation risk of grazing increases, grass is increasingly preferred because it allows the animal to gain energy during its non-grazing time through rumination (Newman et al., 1995).

Godin (1990) also examined the influence of predation risk on diet selectivity, using a dynamic programming model. His approach was similar to ours in that predation risk per time period was greater during prey attack (his ' $\beta_{\mathrm{H}}$ ') than during non-attack actions (' $\beta_{\mathrm{S}}$ '). Because of the apparent similarity between our approach and that of Godin, we will carefully contrast the two models. Godin (1990) presents his model as comparing predator-present and -absent situations. This comparison is made by running his model with two sets of $\beta_{\mathrm{S}}$ and $\beta_{\mathrm{H}}$, one with low values representing a predator-absent situation and one with high values representing a predator-present situation. We will refer to Godin's two scenarios as predator-present and predator-absent (or $\phi=1$ and 0 , respectively).

Godin's model was intended to predict diets of guppies foraging on Daphnia, with and without predators present. He found that guppies 'required significantly more time, on average, to successfully attack, pursue and ingest a large Daphnia than a small one' (Godin, 1990, p. 751). Thus, the attack time values used in the model (obtained from observations of foraging guppies) increased with prey size, and an attack on a larger prey type required that the forager incur the higher predation risk, $\beta_{\mathrm{H}}$, for a longer time. Although $\beta_{\mathrm{H}}$ values were equal for all three prey sizes, this difference in attack time resulted in the three prey having different associated dangers: total danger increased with prey size. Since profitability decreased with prey size, the three prey fell along a negative DP slope. Godin's model predicted an increase in selectivity (increased proportion of small Daphnia in the diet) from predator-absent to predator-present situations, which agrees with the result of our model for such a slope.

However, besides greater values of $\beta_{\mathrm{S}}$ and $\beta_{\mathrm{H}}$ in the predator-present runs than the predatorabsent runs, Godin (1990) also varied two other features of the foraging environment, making it difficult to conclude that the observed effect was due to 'predator presence' alone. First, profitabilities of the three prey classes differed in the two situations, based on measured handling times of guppies. These different profitabilities would have had an influence on diet selectivity independent of that of predator presence. Secondly, the ratio of $\beta_{\mathrm{S}}$ to $\beta_{\mathrm{H}}$ differed between predatorabsent and predator-present situations. Godin used $\beta_{\mathrm{S}}$ and $\beta_{\mathrm{H}}$ values of $1 \times 10^{-6}$ and $2 \times 10^{-6}$, respectively, for the predator-absent run, and $1 \times 10^{-3}$ and $2 \times 10^{-2}$, respectively, for the predatorpresent run. Thus, prey attack was twice as risky as non-attack when no predator was present, but 20 times as risky when a predator was present; no rationale for this difference was provided. Since predation risk equals the product of $\phi$ and the probability of being captured given that a predator is present (danger), this ratio should have remained constant if only the value of $\phi$ changed. This inconsistency may have exaggerated the effect of 'predator presence' on diet selectivity predicted by Godin's model.

An important difference between Godin's (1990) approach and ours is the state variable approach used in our dynamic programming model to simulate the dynamics of $\phi(t)$. In Godin's model, the predation risks (of prey attack and non-attack) have low values when simulating the absence of predators, and high values when simulating predator presence. It may appear that this formulation is reasonable for the experimental conditions that Godin was simulating (in which treatments either had predators present and in full view of subjects, or no predators present). However, since the predation risk parameters are fixed, Godin's model essentially represents a forager who knows that predators either will never arrive in the future ('predator absent') or will never leave ('predator present'). Such certainty is unlikely in nature, and no foragers are likely to 
have been designed with such knowledge. Instead, uncertainty of the type modelled here is more likely to be the rule, and a state-variable approach is likely to be more generally applicable.

\section{Acknowledgements}

Colin Clark, Tom Getty, Bernie Roitberg and Ron Ydenberg provided useful comments on an earlier draft of the manuscript. Tim Rawlings helped with the sculpin experiment. This paper represents one chapter of R.H.'s PhD thesis at Simon Fraser University. Financial support was provided by Natural Sciences and Engineering Research Council (Canada) grant A6869 to L.M.D.

\section{References}

Chesson, J. (1978) Measuring preference in selective predation. Ecology 59, 211-215.

Edmunds, M. (1974) Defence in Animals. Longman, New York.

Gilliam, J.F. (1990) Hunting by the hunted: Optimal prey selection by foragers under predation hazard. In Behavioural Mechanisms of Food Selection (R.N. Hughes, ed.), pp. 797-818. Springer-Verlag, Berlin.

Godin, J.-G.J. (1990) Diet selection under the risk of predation. In Behavioural Mechanisms of Food Selection (R.N. Hughes, ed.), pp. 739-769. Springer-Verlag, Berlin.

Godin, J.-G.J. and Smith, S.A. (1988) A fitness cost of foraging in the guppy. Nature 333, 69-71.

Houtman, R. (1995) The influence of predation risk on within-patch foraging decisions of cryptic animals. $\mathrm{PhD}$ thesis, Simon Fraser University, Burnaby.

Houtman, R. and Dill, L.M. (1994) The influence of substrate color on the alarm response of tidepool sculpins (Oligocottus maculosus; Pisces, Cottidae). Ethology 96, 147-154.

Hugie, D.M., Thuringer, P.L. and Smith, R.J.F. (1991) The response of the tidepool sculpin, Oligocottus maculosus, to chemical stimuli from injured conspecifics, alarm signalling in the Cottidae (Pisces). Ethology 89, 322-334.

Ibrahim, A.A. and Huntingford, F.A. (1989) Laboratory and field studies of the effect of predation risk on foraging in three-spined sticklebacks (Gasterosteus aculeatus). Behaviour 109, 46-57.

Lima, S.L. (1988) Vigilance and diet selection: A simple example in the dark-eyed junco. Can. J. Zool. 66, 593596.

Lima, S.L. and Dill, L.M. (1990) Behavioral decisions made under the risk of predation: A review and prospectus. Can. J. Zool. 68, 619-640.

Mangel, M. and Clark, C.W. (1988) Dynamic Modeling in Behavioral Ecology. Princeton University Press, Princeton, NJ.

Milinski, M. (1984) A predator's costs of overcoming the confusion-effect of swarming prey. Anim. Behav. 32, $1157-1162$.

Morey, S.R. (1990) Microhabitat selection and predation in the Pacific treefrog, Pseudacris regilla. J. Herpetol. 24, 292-296.

Newman, J.A., Parsons, A.J., Thornley, J.H.M., Penning, P.D. and Krebs, J.R. (1995) Optimal diet selection by a generalist grazing herbivore. Funct. Ecol. 9, 255-268.

Stephens, D.W. and Krebs, J.R. (1986) Foraging Theory. Princeton University Press, Princeton, NJ.

\section{Appendix: The dynamic programming equation}

In our dynamic programming model, a forager's fitness at energy state $E$, perceived probability that a predator is present state $(\phi)$ and time $t$ is calculated according to the equation: 


$$
F(E, \phi, t, T)=\sum_{i=0}^{8} \lambda_{i} \operatorname{MAX}\left\{\begin{array}{c}
\left(1-\beta_{0}\right) \sum_{j=0}^{1} D_{j} \times F\left(E(t)-1, \phi_{j}, t+\tau_{0}, T\right) \\
\text { OR } \\
\left(1-\beta_{i}\right) \sum_{j=0}^{1} D_{j} \times F\left(E(t)+Y_{i}, \phi_{j}, t+\tau_{i}, T\right)
\end{array}\right\}
$$

The forager's fitness equals the probability of surviving to the time horizon, $T$, given its current state and time. This probability equals the sum of the survival probabilities for each of nine possible encounter types (indexed as $i$ ) which can occur in any time period, weighted by the probabilities of those encounter types $\left(\lambda_{\mathrm{i}}\right)$. The nine encounter types are: no prey item encountered $(i=0)$, prey type I encountered at one of four attack times $(i=1-4)$, or prey type II encountered at one of four attack times $(i=5-8)$.

For any encounter type, the probability of surviving from $t$ to $T$ is given by the term inside the maximization (MAX). The top and bottom terms inside the large brackets represent the survival probabilities for rejecting and attacking an encountered prey item, respectively. The survival probability for a particular encounter type equals the larger of these two survival probabilities.

The survival probability of either rejecting or attacking an encountered prey item equals the product of the probability of surviving the action $(t$ to $t+\tau)$ and the time span $t+\tau$ to $T$ (where $\tau$ is the duration of the action). The only way of dying in time period $t$ to $t+\tau$ is by predation. Therefore, the probability of surviving time period $t$ to $t+\tau$ equals 1 minus the probability of predation $(\beta)$. The value of $\beta$ is greater for prey captures $\left(\beta_{i}\right)$ than for not capturing prey $\left(\beta_{0}\right)$.

The probability of surviving from $t+\tau$ to $T$ is given by the remaining terms, which have the general form:

$$
\sum_{j=0}^{1} D_{j} \times F\left(E(t)+x, \phi_{j}, t+\tau, T\right)
$$

After any action, the forager scans for predators and either detects a predator $(j=1)$ or not $(j=0)$. The probability that the forager detects a predator, $D_{1}$, equals the product of the $\operatorname{Pr}$ (forager detects predator | predator present) and the $\phi$ value which applies when the forager scans. The forager does not detect a predator with probability $D_{0}=1-D_{1}$. The probability of surviving from $t+\tau$ to $T$ equals the sum of the probabilities of surviving that span if a predator is detected and if no predator is detected, weighted by the probabilities of these two outcomes.

Finally, $F\left(E(t)+x, \phi_{j}, t+\tau, T\right)$ represents the probability of surviving from $t+\tau$ to $T$, given outcome $j$. In this function, $x$ is the change in energy state resulting from the chosen action (if no prey item is attacked, $x=-1$; if a prey item is attacked, $x=$ the net energy gain from the prey item) and $\phi_{j}$ is the value of $\phi$ given outcome $j$. The duration of no prey attack $\left(\tau_{0}\right)$ equals 1 time period and that of prey attack $\left(\tau_{i}\right)$ equals the prey attack time (Table 1). Since the dynamic program is solved backwards through time, the values of this function at $t+\tau$, required to calculate the current $F(E, \phi, t, T)$, have previously been calculated. 